\title{
Shielding Properties of Conductive Plastic Housings Loaded with Printed Circuit Boards
}

\author{
ANTONIO JOSÉ LOZANO-GUERRERO, ${ }^{1}$ MARTIN PAUL ROBINSON, ${ }^{2}$ \\ ALEJANDRO DÍAZ-MORCILLO, ${ }^{1}$ JUAN MONZÓ-CABRERA, ${ }^{1}$ FRANCISCO \\ JAVIER CLEMENTE-FERNÁNDEZ, ${ }^{1}$ and JUAN VICENTE BALBASTRE-
}

TEJEDOR $^{3}$
${ }^{1}$ Departamento de Tecnologías de la Información y las Comunicaciones, Universidad
Politécnica de Cartagena. Cartagena, Spain
${ }^{2}$ Department of Electronics, The University of York. York, UK
${ }^{3}$ Instituto Itaca, Universidad Politécnica de Valencia. Valencia, Spain

\begin{abstract}
In this paper the benefits of using conductive plastics in enclosure configurations have been evaluated taking into account the influence of internal printed circuit boards. Conductive plastic enclosures are a very attractive alternative to traditional metallic enclosures to protect electronic systems against electromagnetic interference. A wide range of conductivities can be obtained with these materials to satisfy a required design. An equivalent model for the printed circuit board has been used to evaluate the effects of radiated interference upon electronic equipment under two orientations. The possibilities and drawbacks of this equivalent model are also discussed. Two different designs with these materials have been studied to analyse the advantages derived from its use with the help of measurements and simulations. Shielding effectiveness and Q-factor have been used to compare the shielding properties of these enclosures with similarly sized metallic ones, taking into account the influence of an internal printed circuit board.
\end{abstract}

Keywords conductive plastic; enclosure; printed circuit board; radiated immunity; shielding effectiveness 


\section{Introduction}

Conductive plastic enclosures for shielding purposes to protect electronic devices from unwanted electromagnetic radiation are being manufactured with plastic materials. However little work has been done regarding the study of their shielding properties and possibilities in enclosure configurations with internal printed circuit boards (PCBs). A wide range of available conductivities may help to obtain the shielding effectiveness (SE) requirements for these housings. Additionally, the minima at certain frequencies in the SE ratio are related to the physical dimensions of the cabinet, and may therefore be avoided with an optimized structure. SE of metallic enclosures has been intensively analysed by using different approaches. Although analytical solutions (Robinson et al., 1998; Azaro et al., 2001) have been provided, numerical methods become necessary when enclosures with non-canonical shapes, internal contents and an arbitrary distribution of surface apertures on the box have to be analysed. Transmission Line Matrix (TLM) (Marvin et al., 2004), Electromagnetic Topology and Mode Matching compared with Finite-Difference Time-Domain (FDTD) (Park et al., 2009), and the Method of Moments (MoM) (Olyslager et al., 1999) are some examples of these numerical methods.

Representation of inner elements in the enclosure such as PCBs becomes a crucial aspect in the susceptibility/emission problem. Loading the enclosure with these devices that must be protected against electromagnetic interference will lead to a completely different electromagnetic problem. Part of the coupled energy will be dissipated by the electronic circuitry, allowing the possibility of a failure in the functions of the systems. Owing to the complexity of the most common type of electronic components grouping, the PCB, a simplification may therefore be needed to enable electromagnetic numerical simulations. Different approaches have been suggested. The simplest one models the 
PCB as a metallic plate (Azaro et al., 2000). Transmission and reflection coefficients have been suggested for a conductive sheet (Robinson et al., 2000) for a set of different PCBs. The inclusion of a dielectric slab next to a metallic plate has been proposed to take into account the losses due to the components and circuitry in the PCB, the materials that are used in the manufacturing process and the grounding configuration (Lozano et al., 2008; Thomas et al., 2001).

The effect of resonance suppression studied analytically in (Yamane et al., 2000) for a double spherical shell with no apertures in its surface has been carried out numerically (Lozano et al., 2005; Lozano et al. 2007) for a rectangular enclosure with an aperture. The experimental verification for empty enclosures of the resonance suppression phenomenon is shown in Lozano et al. (2010) with a multilayer enclosure manufactured with a commercial plastic compound.

In this paper two configurations including conductive plastics and PCB models have been evaluated as a tool for suppressing resonances: (i) a hybrid solution with an outer metallic layer and an inner conductive dielectric layer and (ii) a conductive dielectric enclosure. The PCB model developed in Lozano et al. (2008) has been used for two orientations of the PCB in this work. Inclusion of PCBs allows a more realistic evaluation of the studied shielding structures as the protection of electronic components is the final goal of the enclosures.

Electromagnetic shielding properties are evaluated for an enclosure with an aperture and an inner coating, as depicted in Figure 1, both numerically and experimentally. This enclosure will include a PCB. The metallic layer will be later removed to obtain the shielding levels provided by the conductive plastic enclosure alone. 


\section{Theory}

The classical definition of SE has been used to study the properties of conductive plastics in a shielding enclosure configuration loaded with a PCB. SE can be obtained as the ratio of the field in the selected position in the absence of the shield $\vec{E}_{i}$ and the field at the same location including the shield $\vec{E}_{t}$. It is usually expressed in logarithmic units:

$$
\mathrm{SE}(\mathrm{dB})=20 \log _{10}\left(\frac{\left|\vec{E}_{i}\right|}{\left|\vec{E}_{t}\right|}\right) .
$$

The skin depth parameter, $\delta$, has also a special interest since the design of the inner conductive plastic layer follows the criterion established in Yamane et al. (2000) and Lozano et al. (2007) where the optimum conductivity verifies the ratio thickness $/ \delta \approx 1.15$ for suppressing a selected resonance. $\delta$ is a parameter that shows the penetration level of a wave in a medium and it can be calculated for the general case as

$$
\delta=\frac{1}{\omega \sqrt{\frac{\mu \varepsilon}{2}\left(\sqrt{1+\left(\frac{\sigma}{\omega \varepsilon}\right)^{2}-1}\right)}}
$$

where $\varepsilon=\varepsilon_{r}^{\prime} \varepsilon_{0}$ being $\varepsilon_{r}^{\prime}$ the dielectric constant and $\varepsilon_{0}$ and $\mu_{0}$ the free space permittivity and permeability. $\mu \approx \mu_{0}$ is the magnetic permeability and $\omega=2 \pi f$ where $f$ is the frequency under study. $\sigma$ is the electric conductivity. For $\sigma>>\omega \varepsilon$ the well-known approximation

$$
\delta=\sqrt{\frac{\omega \sigma \mu}{2}}
$$

applies. Skin depth values associated to metallic materials are much smaller than the thickness of any practical metallic enclosure at radio frequencies. Lower conductivities will decrease the shielding capabilities of an enclosure, but as shielding housings due to the input/output interfaces and ventilation requirements for the electronic equipment 
have apertures on their surfaces the shielding levels of the enclosures decrease drastically. In this scenario 30 or $40 \mathrm{~dB}$ can be considered a good protection level for these applications.

\section{Set up}

A $30 \times 12 \times 30 \mathrm{~cm}^{3}$ enclosure has been used for the experiments. The coupling of energy from the outer to the inner part of the cabinet is allowed by using a $10 \times 0.5 \mathrm{~cm}^{2}$ aperture in the centre of the front face. A four layer structure has been specifically designed with sheets made from polystyrene and carbon filler (GoodFellow $\left.{ }^{\circledR}\right)$ with two different thicknesses of $0.1 \mathrm{~cm}$ and $0.3 \mathrm{~cm}$ for the conductive plastic study. Its commercial availability was the reason for its use. A $0.3+0.3+0.1+0.1 \mathrm{~cm}$ sequence of layers fits exactly inside the structure of the box. Special care has been taken with the discontinuities avoiding air gaps between layers that could affect the measurements. An equivalent layer with a $0.8 \mathrm{~cm}$ thickness can be then studied by using the complete set of sheets. A PCB has been placed inside the enclosure to analyse its influence in the SE curve.

An anechoic chamber was used to carry out the measurements using a log-periodic antenna in the range $30 \mathrm{MHz}-2000 \mathrm{MHz}$ to transmit the signal. The antenna was placed $3 \mathrm{~m}$ away from the box. A $4 \mathrm{~cm}$ long receiving monopole was placed in the centre of the top plate inside the enclosure. The reference value for the SE measurement was taken with the metallic plate containing the monopole and the PCB with the help of polystyrene supports. The shield was then added to the structure and the transmitted field measured inside the box.

CST Microwave Studio ${ }^{\mathrm{TM}}$ commercial software that works with the Finite Integration Technique (FIT) has been used for the simulations using a vertical plane wave incidence. 
The conductive plastic properties have been obtained with an impedance/material analyser (Agilent HP4291A RF). Results for $1 \mathrm{GHz}$ frequency have been selected to characterise the plastic structure $\left(\varepsilon_{r}^{\prime}=15\right.$ and $\left.\sigma=0.39 \mathrm{~S} / \mathrm{m}\right)$. This conductivity value is within the limits given by the manufacturer and has been checked inversely with the aid of the simulation tool employed and the measurements. The thickness $t$ has been increased to provide reasonable resonance suppression since this material is not intended for electromagnetic shielding purposes. Owing to the symmetry of the PCB model four inner points have been used to obtain the shielding effectiveness curve inside the equivalent (Figure 2). Two different orientations for the PCB (Figures 3 and 4) have been analysed. Dimensions for the PCB are $13.7 \times 7.8 \mathrm{~cm}^{2}$. The technique described in Lozano et al. (2008) has been used to model the PCB for different orientations as indicated in Table 1 with equivalent values of thickness $d$, dielectric constant and conductivity. This will provide an approximation of the field levels that the electronic components are exposed to. The PCB is unpowered and placed in a floating configuration as in Thomas et al. (2001). The ground plane and the tracks are placed on one side and the electronic components on the other side.

\section{Results}

In Figure 5 the observation points inside the equivalent PCB (a) show how the shielding levels are higher with the conductive plastic. The first resonance has been dampened and SE levels for higher frequencies increase generally. This is due to the absorbing effect of the coating. Energy that was dissipated by the PCB is now absorbed by the coating. A comparison for the simulation and measurement (b) of the studied structure with and without conductive plastic, carried out at the location of the monopole, shows good agreement taking into account that an equivalent model for the PCB has been introduced into the simulations and that the conductive plastic is modelled by the 
measured values of its material properties at $1 \mathrm{GHz}$. Shielding levels have also improved at the monopole for the whole frequency range.

Figure 6 shows the results obtained when the PCB is placed as shown in Figure 4 (scenario 2). The PCB alters the internal electric field spatial distribution and consequently this leads to a completely different SE curve. For the observation points results obtained show how shielding levels have increased once again. Figure 6 b) also shows that the use of a PCB equivalent model and the conductive plastic materials produces simulations that are in good agreement with the measurements. An important drawback of the equivalent model of the PCB can be observed in Figure $6 \mathrm{~b}$ ) for the empty metallic case (left) where at the frequency of $750 \mathrm{MHz}$ the simulation overestimates the real value of the SE. This is due to the absorbing properties of the equivalent model that was obtained in a closed scenario with two exciting probes (Lozano et al., 2008). This scenario differs from the open plane wave configuration employed in this paper. For the plastic loaded case Figure $6 \mathrm{~b}$ ) (right) the equivalent model works much better as the conductive dielectric layer is the main factor responsible for the absorption of the energy.

Table 2 shows measured and simulated Q-factors and resonant frequencies obtained for the first resonance in Figures 5 (b) and 6 (b). Benefits of the coating can be clearly appreciated for scenario 1, however results for scenario 2 with and without coating seem not to provide this large reduction in the Q-factors. Shielding levels however are around $20 \mathrm{~dB}$ so there is less need to provide better results for this case. Measurements and simulations show good agreement once again.

Figure 7 shows the optimized results $(f=700 \mathrm{MHz}$ and $\sigma=120 \mathrm{~S} / \mathrm{m})$ for the hybrid structure (outer metallic layer + inner coating) for scenario 1 that maximize the reflection loss of the conductive plastic layer. $\varepsilon_{r}^{\prime}=2$ has been introduced in the 
simulations for the coating as the optimum not depends on this value (Lozano et al., (2007)) when the good conductor approximation (Eq. (3)) is assumed. Similar benefits can be observed as in the case of $0.8 \mathrm{~cm}$ commercial sample coating, for a layer only 0.2 cm thick. A metallic painting or a metallic enclosure could be used to provide the metallic protection in a hybrid enclosure. Unfortunately scratches could decrease the shielding properties of the conductive plastic cabinet in the case of a painted layer. Removing the metallic layer leads to the same results as the conductive dielectric structure.

The shielding levels obtained for the lower frequencies are the main noticeable difference as can be seen in Figure 7. However values higher than $30 \mathrm{~dB}$ may be valid for a good protection. Nevertheless higher conductivities may provide better SE levels depending upon the design requirements if needed.

Figure 8 shows similar results for the $0.2 \mathrm{~cm}$ optimized structure for scenario 2 . Shielding levels have decreased for frequencies below the first resonant frequency, owing to the conductivity reduction from the PEC case to a finite $120 \mathrm{~S} / \mathrm{m}$ value in the case of the conductive plastic. However good shielding values around $30 \mathrm{~dB}$ are achieved at the lower frequencies. Both the hybrid and the conductive dielectric case shown in Figures 7 and 8 have a lossy layer with a $120 \mathrm{~S} / \mathrm{m}$ conductivity and $0.2 \mathrm{~cm}$ thickness. This layer allows dampened resonances and a general increase in the shielding levels at higher frequencies when comparing to the results of the PEC cavity depicted in figures 5 and 6 b) left for both scenarios respectively. However at lower frequencies the conductive dielectric case shows reduced shielding levels than the PEC and the hybrid structure.

Table 3 shows Q-factors obtained from the half-power points of the first resonant frequency, seen in Figures 7 and 8 . Slightly better results can be observed for the 
conductive plastic case.

\section{Conclusion}

In this paper the benefits and possibilities of conductive plastics have been evaluated taking into account the loading of the cabinet. Simulations and measurements have been carried out including an internal PCB to compare the shielding effectiveness levels of structures with conductive plastics with those of traditional metallic cabinets.

An equivalent model for the PCB has been included for the simulations showing good behaviour with measurements. It has been demonstrated however that care must be taken when using it as it may produce an overestimation of the SE curve at certain frequencies.

Resonance suppression provided at the resonance minima associated with the dimensions of the enclosure, and the general increase of shielding levels at higher frequencies, are the two main advantages of both structures. These features have been verified experimentally and numerically by obtaining SE curves and evaluating the Qfactor of at least the first resonance appearing in the frequency response.

Designs will vary depending upon the suppression frequency. Higher frequencies will require lower conductivities of conductive plastics, and shielding levels for lower frequencies will decrease. Higher conductivities will increase shielding levels at lower frequencies, however, the resonance suppression level may be not as good as the optimum value. Finally it must be pointed out that results obtained for the hybrid solution may not be optimum for the conductive dielectric case.

\section{Acknowledgement}

This work was done partly during a research stay funded by the "Programa de formación y movilidad del personal docente e investigador de la Universidad 
Politécnica de Cartagena (PMPDI-UPCT-2007)" at the Physical Layer Group, Department of Electronics, The University of York.

\section{References}

Azaro, R., R. S. Caorsi, M. Cosso, G. M. Costini, M. Donelli, R. Ene, G. L. Gragnani \& M. Pastorino. 2000. A semianalythical approach for the evaluation of radiated immunity on a printed-circuit board in metallic enclosures. Microwave. Opt. Technol. Lett. 27: 204-207.

Azaro, R., R., S. Caorsi, M. Donelli \& G. L. Gragnani. 2001. Evaluation of the effects of an external incident wave on metallic enclosures with rectangular apertures. Microwave. Opt. Technol. Lett. 28: 289-293.

Lozano-Guerrero, A. J., A. Díaz, J. V. Balbastre. 2007. Resonance suppression in enclosures with a metallic-lossy dielectric layer by means of genetic algorithms. IEEE International Symposium on Electromagnetic Compatibility. Hawaii, USA. 8-13. July.

Lozano-Guerrero, A. J., A. Díaz, J. V. Balbastre, L. Nuño, A. B. Calvo \& J. Pitarch. 2005. Damping of resonances in a metallic enclosure through conductive polymers. $35^{\text {th }}$ European Microwave Conference. Paris, France. 4-6 October.

Lozano-Guerrero, A. J., M. P. Robinson, A. Diaz-Morcillo \& J. V. Balbastre. 2008. Evaluation and optimization of an equivalent model for printed circuit boards inside metallic enclosures. General Assembly URSI. Chicago, USA. 7-16 August. 
Lozano-Guerrero, A. J., M. P. Robinson, A. Diaz-Morcillo \& J. V. Balbastre. 2010. Benefits of using conductive plastics in shielding configurations to reduce radiated electromagnetic interference. Microwave. Opt. Technol. Lett. 52: 2476-2480.

Marvin, A. C., J. F. Dawson, S. Ward, L. Dawson, J. Clegg \& A. Weisenfeld. 2004. A proposed new definition and measurement of the shielding effect of equipment enclosures. IEEE Trans. Electromag. Compat. 46: 459-468.

Olyslager, F., E. Laermans, D. D. Zutter, S. Criel, R. D. Smedt, N. Lietaert \& A. D. Clercq. 1999. Numerical and experimental study of the shielding effectiveness of a metallic enclosure. IEEE Trans. Electromag. Compat. 41: 202-212.

Park, Y-M., Y. Lee, Y. S. Chung, C. Cheon \& H-K Jung. 2009. Electromagnetic field penetration analysis of a rectangular aperture-backed cavity based on combination of electromagnetic topology and mode matching. Electromagnetics. 29:447-462.

Robinson, M. P., S. J. Porter \& P Op gen Oorth. 2000. Reflection and transmission coefficients of printed circuit boards. $4^{\text {th }}$ Eur. Symp. EMC. Brugge, Belgium. September.

Robinson, M. P., T. M. Benson, C. Christopoulos, J. F. Dawson, M. C. Ganley, A. C. Marvin, S. J. Porter \& D. W.P. Thomas. 1998. Analytical formulation for the shielding effectiveness of enclosures with apertures. IEEE Trans. Electromag. Compat. 40: 240248. 
Thomas, D. W. P., A. C. Denton, T. Konefal, T. Benson, C. Christopoulos, J. F. Dawson, A. Marvin, S. J. Porter \& P. Sewell. 2001. Model of the electromagnetic fields inside a cuboidal enclosure populated with conducting planes or printed circuit boards. IEEE Trans. Electromag. Compat. 43: 161-169.

Yamane, T., A. Nishikata \& Y Shimizu. 2000. Resonance suppression of a spherical electromagnetic shielding enclosure by using conductive dielectrics. IEEE Trans. Electromag. Compat. 42: 441-448. 


\section{List of Captions}

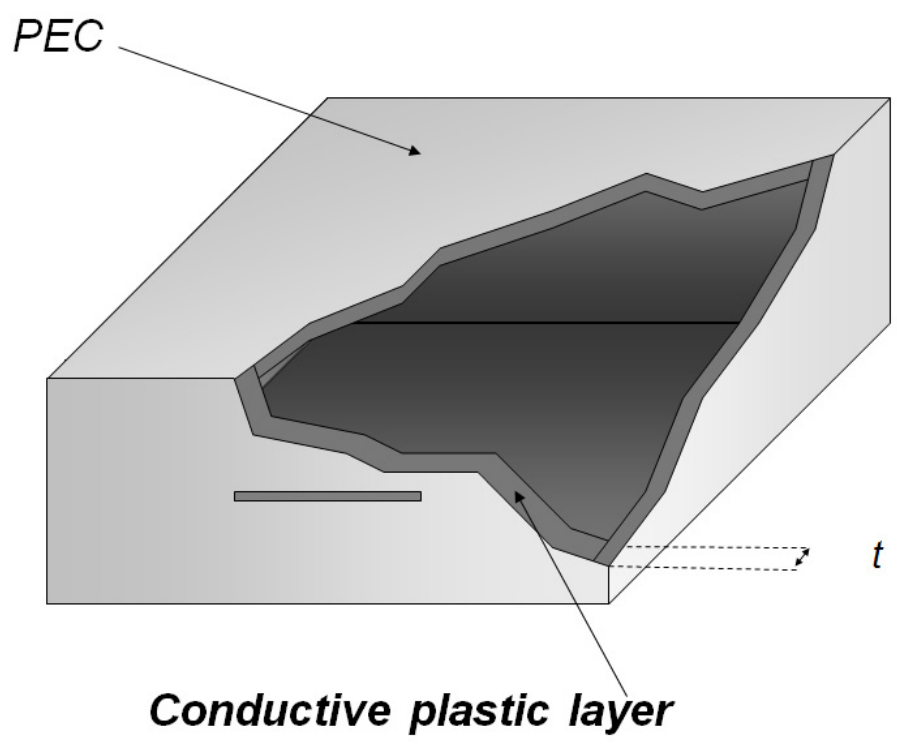

Figure 1 Studied enclosure with an external perfectly electrically conductive (PEC) layer and an inner conductive layer of thickness $t$. 


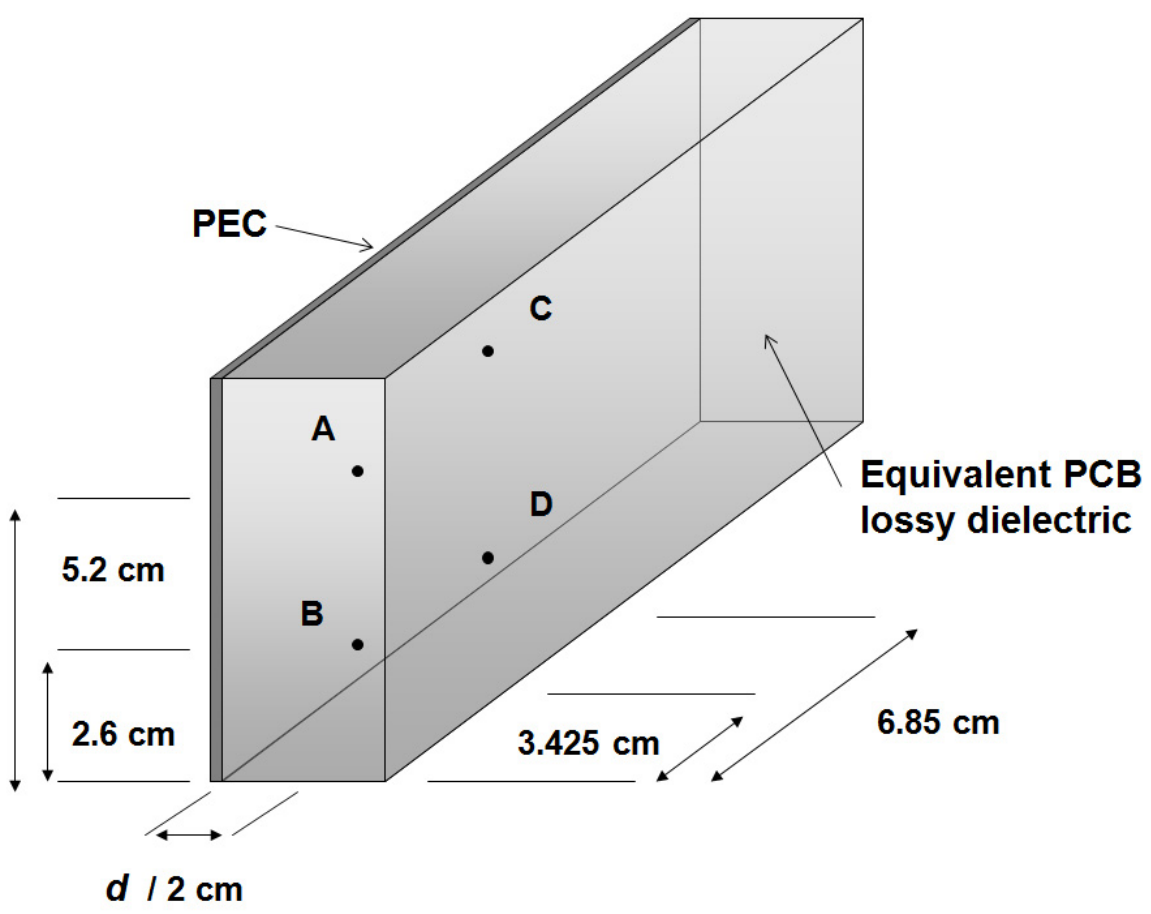

Figure 2 PCB model and evaluation points. 
Shielding Properties of Conductive Plastic Housings with PCBs

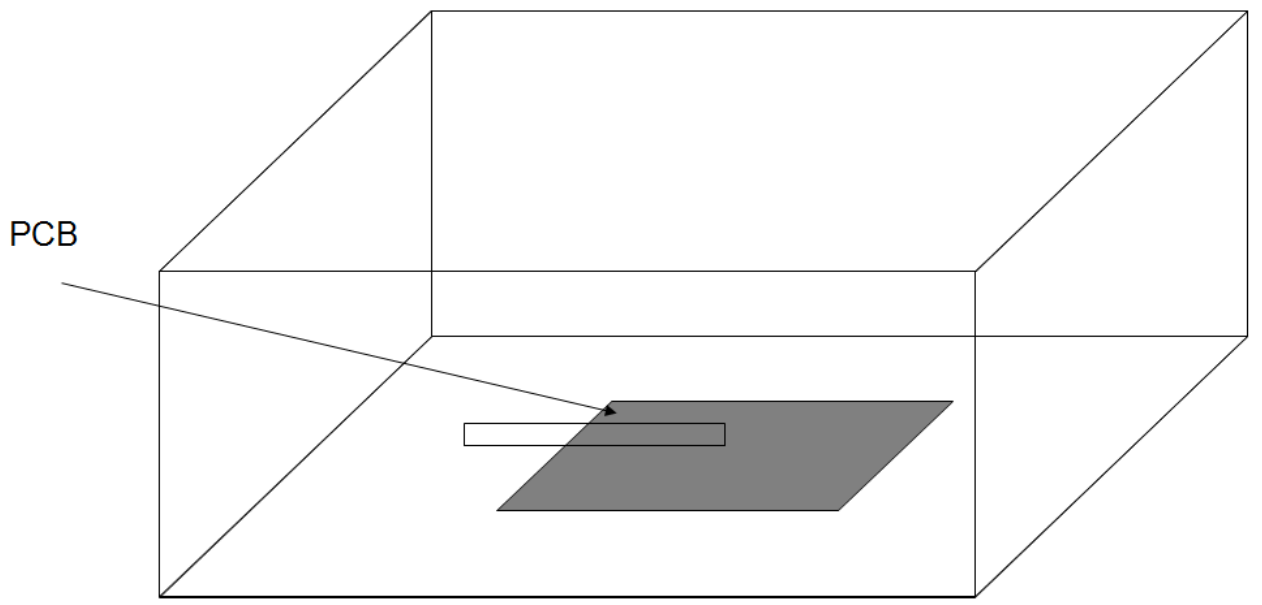


Figure $3 \mathrm{PCB}$ on the floor of the enclosure (scenario 1).

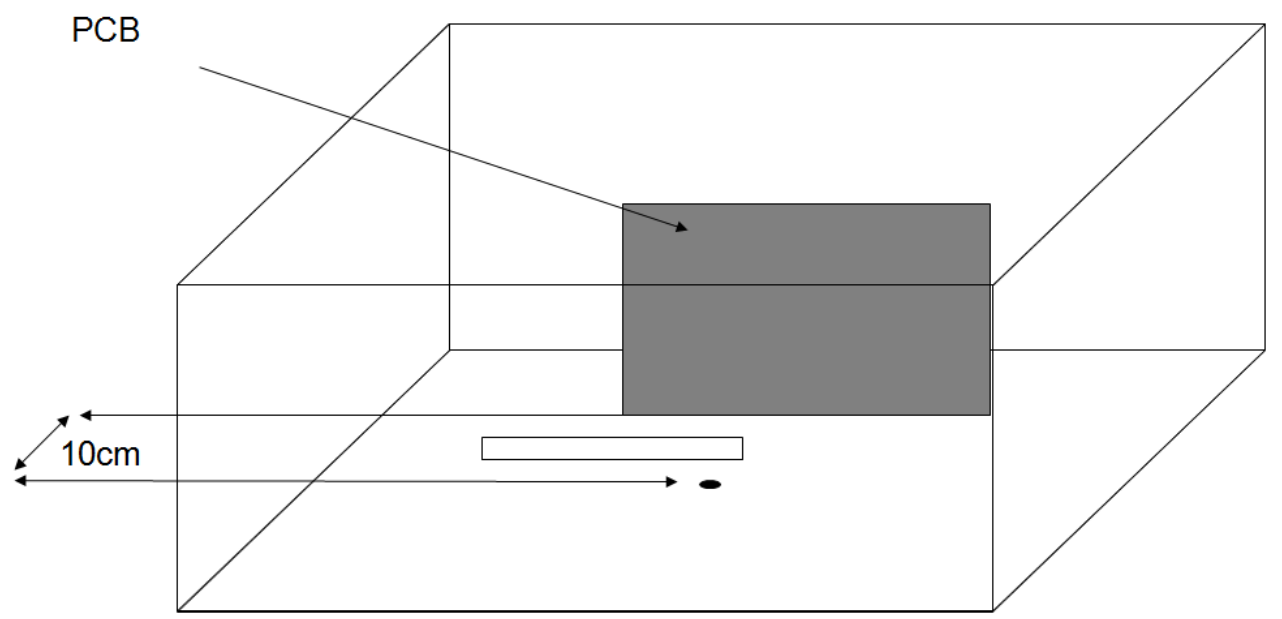

Figure 4 PCB standing parallel to the aperture (scenario 2). 


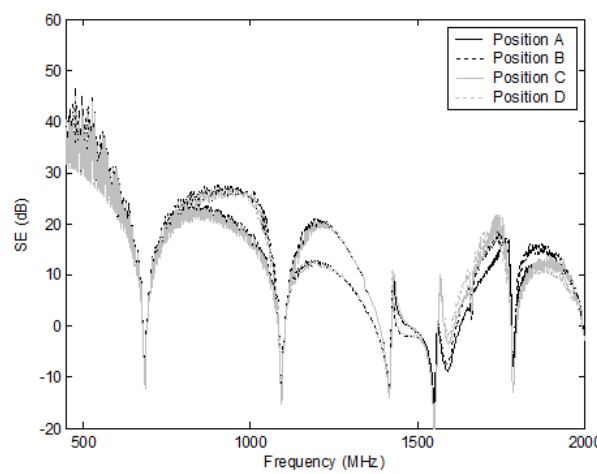

a)

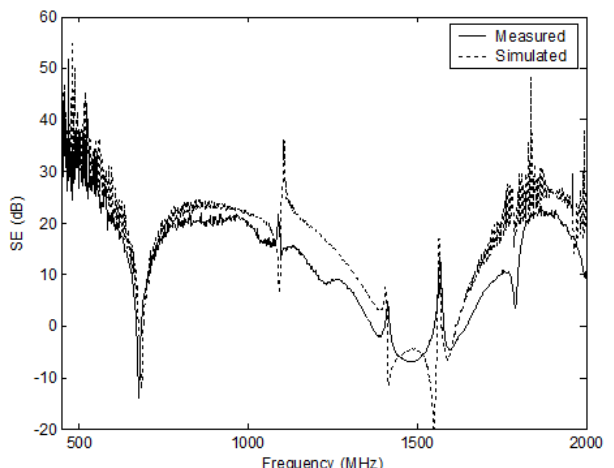

b)

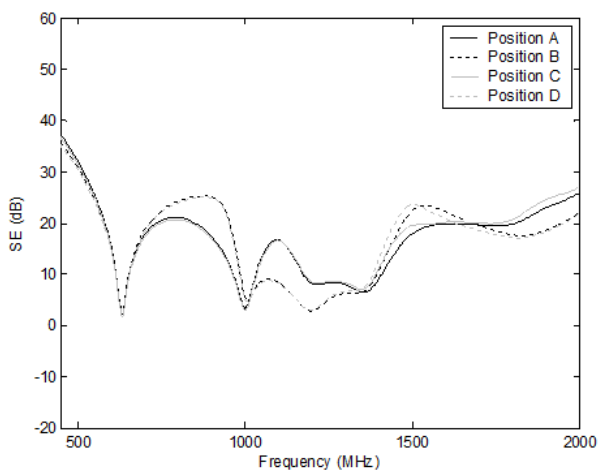

a)

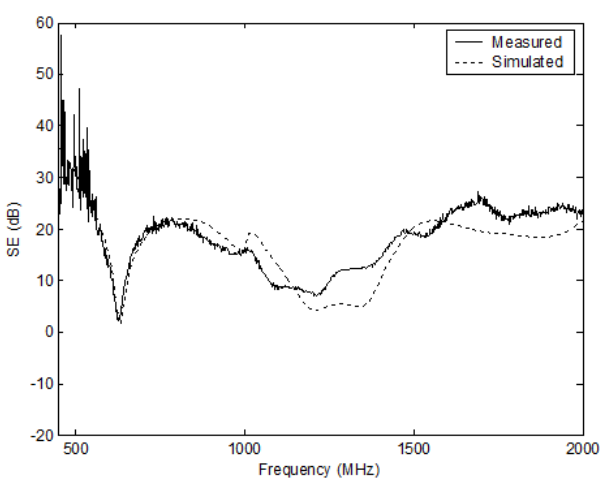

b)

Figure 5 Results for scenario 1 with (right) and without (left) conductive plastic (0.8 $\mathrm{cm}$ ). a) simulation at the PCB model evaluation points, b) comparison of measurements and simulation at the centre of the monopole location (ceiling) (y-component of Efield). 


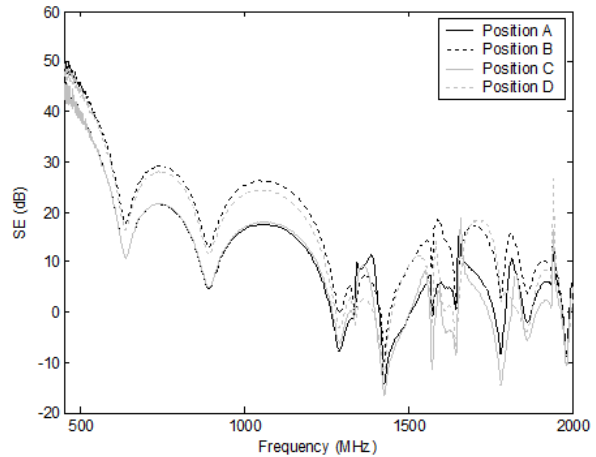

a)

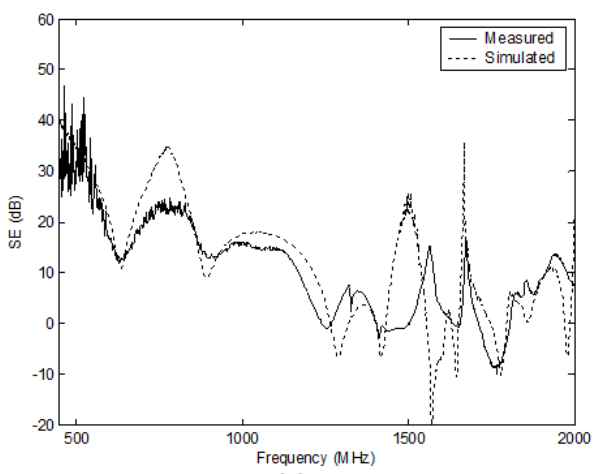

b)

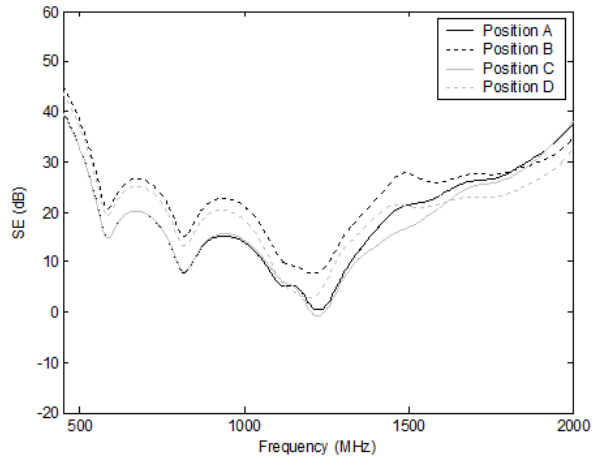

a)

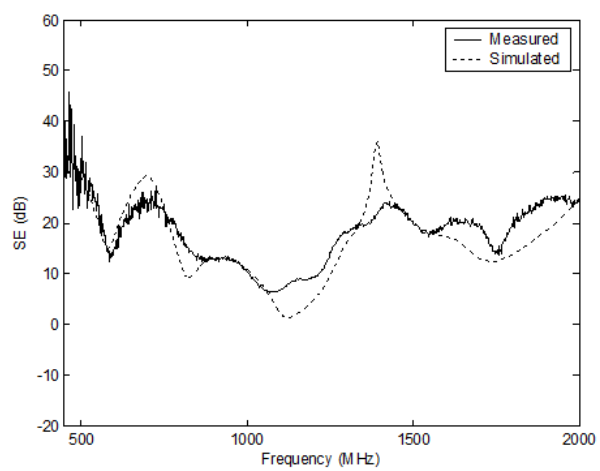

b)

Figure 6 Results for scenario 2 with (right) and without (left) conductive plastic (0.8 $\mathrm{cm})$. a) simulated SE at PCB evaluation points, b) comparison of measurements and simulation at the centre of the monopole location (ceiling) ( $y$-component of E-field). 
Shielding Properties of Conductive Plastic Housings with PCBs

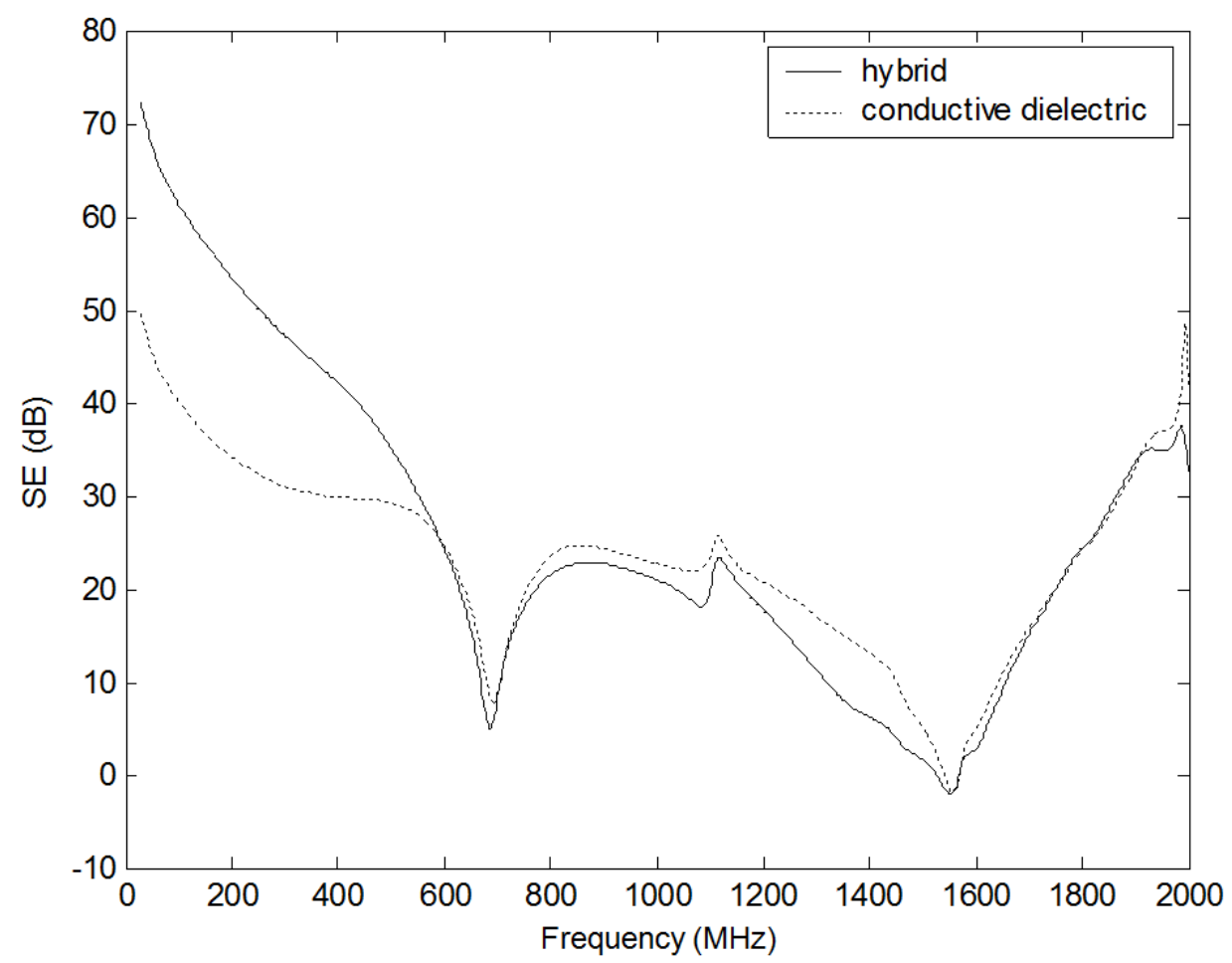

Figure 7 Optimized results for a $0.2 \mathrm{~cm}$ hybrid structure for scenario 1 and structure removing the outer PEC layer. Simulation ( $y$ - component of E-field). 
Shielding Properties of Conductive Plastic Housings with PCBs

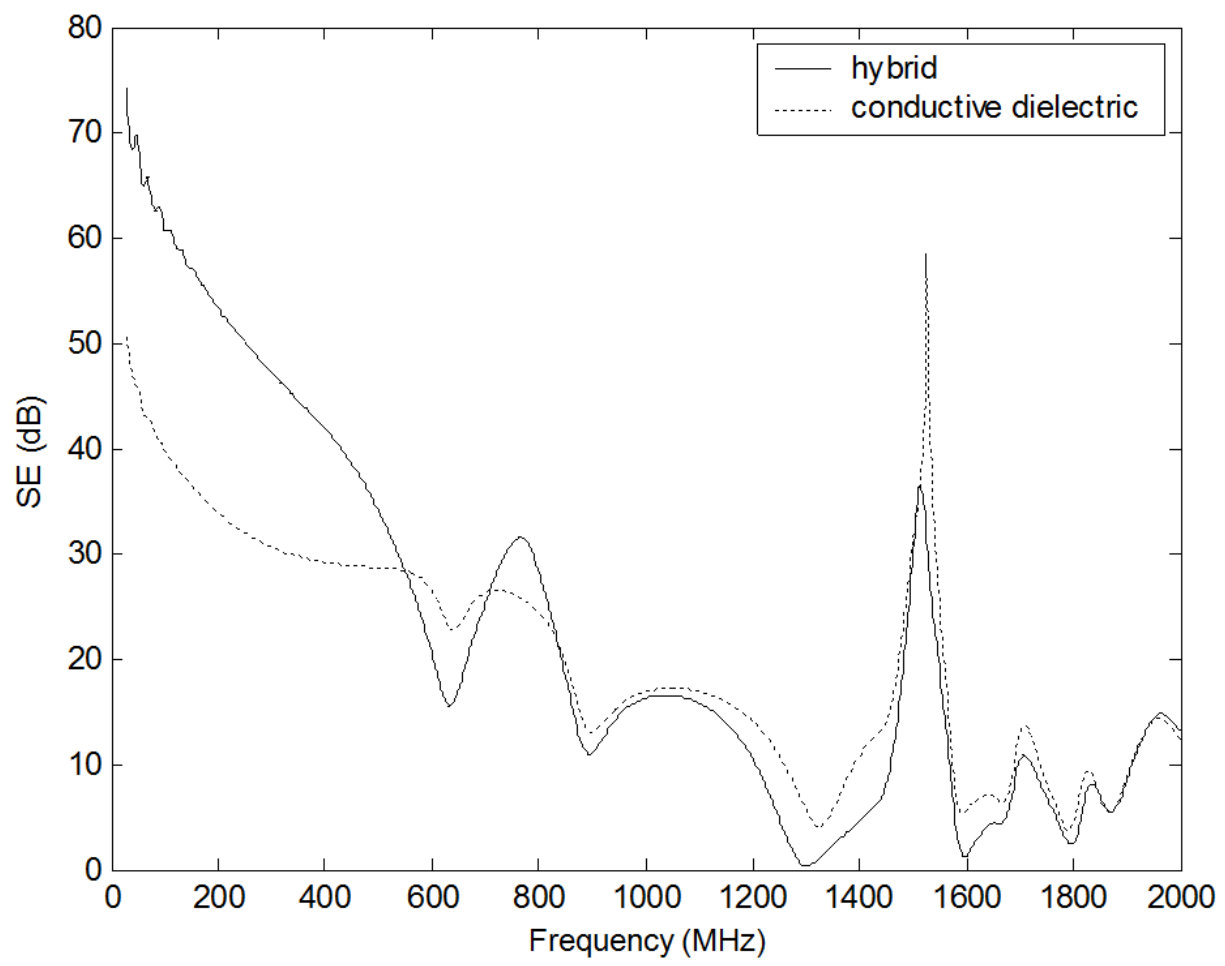

Figure 8 Optimized results for a $0.2 \mathrm{~cm}$ hybrid structure for scenario 2 and structure removing the outer PEC layer. Simulation ( $y$-component of E-field). 


\section{List of Tables}

Table 1

Equivalent $\mathrm{PCB}$ properties

\begin{tabular}{cccc}
\hline Set up & Thickness $d(\mathrm{~cm})$ & $\varepsilon_{r}^{\prime}$ & $\sigma(\mathrm{S} / \mathrm{m})$ \\
\hline Fig. 3 & 0.88 & 4.19 & 0.036 \\
Fig. 4 & 0.68 & 1.56 & 0.096 \\
\hline
\end{tabular}

Table 2

First resonance frequency and Q-factor. Measured $(m)$ and simulated $(s)$

\begin{tabular}{ccccc}
\hline Set up & $f_{m}(\mathrm{MHz})$ & $f_{s}(\mathrm{MHz})$ & $Q_{m}$ & $Q_{s}$ \\
\hline 1 & 680.29 & 687.30 & 245.00 & 154.63 \\
$1+$ coat. & 630.55 & 634.21 & 36.58 & 37.50 \\
2 & 636.13 & 637.20 & 9.72 & 24.13 \\
$2+$ coat. & 592.23 & 583.79 & 20.62 & 13.78 \\
\hline
\end{tabular}

Table 3

First resonance frequency and Q-factor of simulated scenarios

\begin{tabular}{ccc}
\hline Set up & $f_{s}(\mathrm{MHz})$ & $Q_{s}$ \\
\hline 1 Hybrid & 686.20 & 28.11 \\
1 Conductive & 692.19 & 23.91 \\
2 Hybrid & 633.88 & 13.49 \\
2 Conductive & 644.29 & 8.03 \\
\hline
\end{tabular}

